\title{
PRINCIPAL FUNCTIONS FOR BI-FREE CENTRAL LIMIT DISTRIBUTIONS
}

\author{
KENNETH J. DYKEMA AND WONHEE NA
}

\begin{abstract}
We find the principal function of the completely non-normal operator $l\left(v_{1}\right)+l\left(v_{1}\right)^{*}+i\left(r\left(v_{2}\right)+\right.$ $\left.r\left(v_{2}\right)^{*}\right)$ on a subspace of the full Fock space $\mathcal{F}(\mathcal{H})$ which arises from a bi-free central limit distribution. As an application, we find the essential spectrum of this operator.
\end{abstract}

\section{Introduction and Preliminaries}

Bi-free independence was introduced by Voiculescu as a generalization of free independence in a noncommutative probability space $(\mathcal{A}, \varphi)$. He considered a two-faced family of non-commutative random variables, $\left(X_{1}, X_{2}\right)$, in $(\mathcal{A}, \varphi)$ and the expectation values for such a combined system of left and right variables. In 7 , Voiculescu proved a bi-free central limit theorem and described the family of distributions that appear as limits. These are called bi-free central limit distributions.

1.1. Bi-freeness. Let $z=\left(\left(z_{i}\right)_{i \in I},\left(z_{j}\right)_{j \in J}\right)$ be a two-faced family in a non-commutative probability space $(\mathcal{A}, \varphi)$ where $I$ and $J$ are disjoint index sets.

Definition $1.1(\sqrt{7})$. The two-faced families $z^{\prime}$ and $z^{\prime \prime}$ are said to be bi-freely independent (abbreviated bi-free $)$ if there exist two vector spaces $\left(\mathcal{X}^{\prime}, \mathcal{X}^{\prime \circ}, \xi^{\prime}\right)$ and $\left(\mathcal{X}^{\prime \prime}, \mathcal{X}^{\prime \prime \prime}, \xi^{\prime \prime}\right)$ with specified state vectors and unital homomorphisms $l^{\epsilon}: \mathbb{C}\left\langle z_{i}^{\epsilon} \mid i \in I\right\rangle \rightarrow \mathcal{L}\left(\mathcal{X}^{\epsilon}\right)$ and $r^{\epsilon}: \mathbb{C}\left\langle z_{j}^{\epsilon} \mid j \in J\right\rangle \rightarrow \mathcal{L}\left(\mathcal{X}^{\epsilon}\right)$ with $\epsilon \in\left\{^{\prime}{ }^{\prime \prime},\right\}$ such that the two-faced families $T^{\epsilon}=\left(\left(\lambda^{\epsilon} \circ l^{\epsilon}\left(z_{i}^{\epsilon}\right)\right)_{i \in I},\left(\rho^{\epsilon} \circ r^{\epsilon}\left(z_{j}^{\epsilon}\right)\right)_{j \in J}\right)$ have the same joint distribution in $\left(\mathcal{L}(\mathcal{X}), \varphi_{\xi}\right)$ as $z^{\prime}$ and $z^{\prime \prime}$ where $\left(\mathcal{X}, \mathcal{X}^{\circ}, \xi\right)=\left(\mathcal{X}^{\prime}, \mathcal{X}^{\prime \circ}, \xi^{\prime}\right) *\left(\mathcal{X}^{\prime \prime}, \mathcal{X}^{\prime \prime \circ}, \xi^{\prime \prime}\right)$ and $\lambda^{\epsilon}$ and $\rho^{\epsilon}$ are left and right representations of $\mathcal{L}\left(\mathcal{X}^{\epsilon}\right)$ on $\mathcal{L}(\mathcal{X})$.

Definition 1.2 $(\overline{7]})$. For each map $\alpha:\{1, \ldots, n\} \rightarrow I \amalg J$ there is a unique universal polynomial $R_{\alpha}$ in commuting variables $X_{\alpha\left(k_{1}\right) \cdots \alpha\left(k_{r}\right)}, 1 \leq k_{1}<\cdots<k_{r} \leq n$ such that

(i) $R_{\alpha}$ is homogeneous of degree $n$ where $X_{\alpha\left(k_{1}\right) \cdots \alpha\left(k_{r}\right)}$ is assigned degree $r$,

(ii) the coefficient of $X_{\alpha(1) \cdots \alpha(n)}$ is 1 , and

(iii) if $z^{\prime}=\left(\left(z_{i}^{\prime}\right)_{i \in I},\left(z_{j}^{\prime}\right)_{j \in J}\right)$ and $z^{\prime \prime}=\left(\left(z_{i}^{\prime \prime}\right)_{i \in I},\left(z_{j}^{\prime \prime}\right)_{j \in J}\right)$ are bi-free pairs of two-faced families of noncommutative random variables in $(\mathcal{A}, \varphi)$, then

$$
R_{\alpha}\left(z^{\prime}\right)+R_{\alpha}\left(z^{\prime \prime}\right)=R_{\alpha}\left(z^{\prime}+z^{\prime \prime}\right)
$$

where $R_{\alpha}(z)=R_{\alpha}\left(\varphi\left(z_{\alpha\left(k_{1}\right)} \cdots z_{\alpha\left(k_{r}\right)}\right) \mid 1 \leq k_{1}<\cdots<k_{r} \leq n\right)$.

These polynomials $R_{\alpha}$ are called bi-free cumulants.

Theorem 1.3 ([7]). A two-faced family $z$ of non-commutative random variables has a bi-free central limit distribution if and only if $R_{\alpha}(z)=0$ whenever $\alpha:\{1, \ldots, n\} \rightarrow I \amalg J$ with $n=1$ or $n \geq 3$.

We now recall the notion of a two-faced system with rank $\leq 1$ commutation given in 8 .

Definition 1.4. An implemented non-commutative probability space is a triple $(\mathcal{A}, \varphi, P)$ where $(\mathcal{A}, \varphi)$ is a non-commutative probability space and $P=P^{2} \in \mathcal{A}$ is an idempotent so that

$$
P a P=\varphi(a) P \text { for all } a \in \mathcal{A} .
$$

Date: October 12, 2015.

2000 Mathematics Subject Classification. 46L54 (47A65).

Key words and phrases. Bi-freeness, bi-free central limit distribution, principal function.

Research supported in part by NSF grant DMS-1202660. 
An implemented $C^{*}$-probability space $(\mathcal{A}, \varphi, P)$ will satisfy additional requirements that $(\mathcal{A}, \varphi)$ is a $C^{*}$ probability space and that $P=P^{*}$. If a two-faced family $\left(\left(z_{i}\right)_{i \in I},\left(z_{j}\right)_{j \in J}\right)$ in an implemented noncommutative probability space $(\mathcal{A}, \varphi, P)$ satisfies that

$$
\left[z_{i}, z_{j}\right]=\lambda_{i, j} P \text { for some } \lambda_{i, j} \in \mathbb{C}, i \in I, j \in J,
$$

then the family $\left(\left(z_{i}\right)_{i \in I},\left(z_{j}\right)_{j \in J}\right)$ is called a system with rank $\leq 1$ commutation where $\left(\lambda_{i, j}\right)_{i \in I, j \in J}$ is the coefficient matrix of the system.

Definition 1.5. Let $\mathcal{H}$ be a complex Hilbert space. Then the full Fock space on $\mathcal{H}$ is

$$
\mathcal{F}(\mathcal{H})=\mathbb{C} \Omega \oplus \bigoplus_{n \geq 1} \mathcal{H}^{\otimes n}
$$

where $\Omega$ is called the vacuum vector and has norm one. The vacuum expectation is defined as $\varphi_{\Omega}=\langle\cdot \Omega, \Omega\rangle$ on $\mathcal{F}(\mathcal{H})$. For $\xi \in \mathcal{H}$, the left creation operator $l(\xi) \in B(\mathcal{F}(\mathcal{H}))$ is given by the formulas $l(\xi) \Omega=\xi$ and

$$
l(\xi)\left(\xi_{1} \otimes \cdots \otimes \xi_{n}\right)=\xi \otimes \xi_{1} \otimes \cdots \otimes \xi_{n}
$$

for all $n \geq 1$ and $\xi_{1}, \cdots, \xi_{n} \in \mathcal{H}$. The adjoint $l(\xi)^{*}$ of $l(\xi)$ is called the left annihilation operator. The right creation operator $r(\xi) \in B(\mathcal{F}(\mathcal{H}))$ is determined by the formulas $r(\xi) \Omega=\xi$ and

$$
r(\xi)\left(\xi_{1} \otimes \cdots \otimes \xi_{n}\right)=\xi_{1} \otimes \cdots \otimes \xi_{n} \otimes \xi
$$

for all $n \geq 1$ and $\xi_{1}, \cdots, \xi_{n} \in \mathcal{H}$. The adjoint $r(\xi)^{*}$ of $r(\xi)$ is called the right annihilation operator.

Theorem 1.6 (Theorem 7.4 of 7 ). For each matrix $C=\left(C_{k l}\right)_{k, l \in I \amalg J}$ with complex entries, there is exactly one bi-free central limit distribution $\varphi_{C}: \mathbb{C}\left\langle Z_{k} \mid k \in I \amalg J\right\rangle \rightarrow \mathbb{C}$ so that

$$
\varphi_{C}\left(Z_{k} Z_{l}\right)=C_{k l} \text { for each } k, l \in I \amalg J .
$$

If $h, h^{\prime}: I \amalg J \rightarrow \mathcal{H}$ are maps into the Hilbert space $\mathcal{H}$ and we define

$$
\begin{aligned}
& z_{i}=l(h(i))+l^{*}\left(h^{\prime}(i)\right) \text { if } i \in I \\
& z_{j}=r(h(j))+r^{*}\left(h^{\prime}(j)\right) \text { if } j \in J
\end{aligned}
$$

then $z=\left(\left(z_{i}\right)_{i \in I},\left(z_{j}\right)_{j \in J}\right)$ has a bi-free central limit distribution $\varphi_{C}$ where $C_{k l}=\left\langle h(l), h^{\prime}(k)\right\rangle$. Every bi-free central limit distribution when I and $J$ are finite can be obtained in this way.

Remark 1.7. The bi-free two-faced system in Theorem 1.6 is an example of rank $\leq 1$ commutation. Indeed, $\left(B(\mathcal{F}(\mathcal{H})), \varphi_{\Omega}, P\right)$ is an implemented $C^{*}$-probability space where $\varphi_{\Omega}$ is the vacuum expectation and $P$ is a projection on $\mathbb{C} \Omega$. We have $\left[z_{i}, z_{j}\right]=\left(\left\langle h(j), h^{\prime}(i)\right\rangle-\left\langle h(i), h^{\prime}(j)\right\rangle\right) P$.

1.2. Principal function of a completely non-normal operator. Let $T$ be a completely non-normal operator on a Hilbert space $\mathcal{H}$ with self-commutator $T^{*} T-T T^{*}=-2 C$ that is trace class. Set $U=$ $\frac{1}{2}\left(T+T^{*}\right)$ and $V=-\frac{1}{2} i\left(T-T^{*}\right)$. Consider the $\mathrm{C}^{*}$-algebra generated by $C$ and the identity operator on $\mathcal{H}$; it is isometrically isomorphic to $C(\sigma(C))$, the complex valued continuous functions on $\sigma(C)$, by the Gelfand-Naimark theorem. Consider the function on $\sigma(C)$,

$$
t \mapsto \begin{cases}-i \sqrt{-t}, & t<0 \\ 0, & t=0 \\ \sqrt{t}, & t>0\end{cases}
$$

and there exists the unique element $\hat{C}$ in the $\mathrm{C}^{*}$-algebra corresponding to this function by the Gelfand transform. Note that $\hat{C}^{2}=C$ and $\hat{C} \hat{C}^{*}=\hat{C}^{*} \hat{C}=|C|$.

The determining function of the operator $T$ is defined to be

$$
E(l, s)=I+\frac{1}{i} \hat{C}(V-l)^{-1}(U-s)^{-1} \hat{C}
$$


for $l \in \mathbb{C} \backslash \sigma(V)$ and $s \in \mathbb{C} \backslash \sigma(U)$. Then $E(l, s)$, for each fixed $l$ and $s$, is an invertible element in the $\mathrm{C}^{*}$-algebra generated by $T$ and $I$. Since $\operatorname{det}(I+A B)=\operatorname{det}(I+B A)$ when $A$ is compact with $A B$ and $B A$ in trace class, we have

$$
\begin{aligned}
\operatorname{det} E(l, s) & =\operatorname{det}\left(I+\frac{1}{i} C(V-l)^{-1}(U-s)^{-1}\right) \\
& =\operatorname{det}\left((V-l)(U-s)(V-l)^{-1}(U-s)^{-1}\right) .
\end{aligned}
$$

The principal function $g$ is defined in [4] to be the element of $L_{1}\left(\mathbb{R}^{2}\right)$ such that

$$
\operatorname{det} E(l, s)=\exp \left(\frac{1}{2 \pi i} \iint g(\delta, \gamma) \frac{d \delta}{\delta-l} \frac{d \gamma}{\gamma-s}\right) .
$$

It is known that $\operatorname{supp}(g)$ is contained in $\left\{(\delta, \gamma) \in \mathbb{R}^{2} \mid \gamma+i \delta \in \sigma(T)\right\}$. Moreover, it is a complete unitary invariant for $T$ if $C$ has one dimensional range; that is, two completely non-normal operators $T$ and $T^{\prime}$ are unitarily equivalent if and only if their principal functions agree, assuming each of $T$ and $T^{\prime}$ has a self-commutator with one dimensional range. In Theorem 8.1 of [4, it is proved that

$$
g(\delta, \gamma)=\operatorname{ind}(T-(\gamma+i \delta))
$$

if $\gamma+i \delta$ is not in the essential spectrum $\sigma_{e}(T)$. This result implies that the principal function $g$ of $T$ is an extension of the Fredholm index of $T-z$ to the whole plane. However, it is not the typical situation that $g$ assumes only integer values on the plane; indeed the map $T \mapsto g$ is onto, namely (see [5]), any summable function on $\mathbb{R}^{2}$ with compact support is the principal function of a completely non-normal operator with a trace class self-commutator.

\section{THE PRINCIPAL FUNCTION OF CERTAIN OPERATORS}

2.1. Let $\mathcal{H}$ be a Hilbert space and $v_{1}, v_{2} \in \mathcal{H}$. We consider the operator $T$ on $\mathcal{F}(\mathcal{H})$ given by

$$
T=X_{1}+i X_{2}, \quad \text { with } \quad X_{1}=l\left(v_{1}\right)+l\left(v_{1}\right)^{*}, \quad X_{2}=r\left(v_{2}\right)+r\left(v_{2}\right)^{*} .
$$

This arises from the bi-free central limit distribution and was described in Example 3.10 of [8]. As we discussed in Section 1 , we have $\left[X_{1}, X_{2}\right]=2 i\left(\operatorname{Im}\left\langle v_{2}, v_{1}\right\rangle\right) P$ in the implemented $C^{*}$-probability space $\left(B(\mathcal{F}(\mathcal{H})), \varphi_{\Omega}, P\right)$, so that

$$
\left[T, T^{*}\right]=4\left(\operatorname{Im}\left\langle v_{2}, v_{1}\right\rangle\right) P .
$$

Both the spectrum and the essential spectrum of $X_{1}$ on $\mathcal{F}(\mathcal{H})$ equal $\left[-2\left\|v_{1}\right\|, 2\left\|v_{1}\right\|\right]$ and those of $X_{2}$ equal $\left[-2\left\|v_{2}\right\|, 2\left\|v_{2}\right\|\right]$. By the following easy lemma, which is well known but whose proof we include for convenience, the spectrum of the operator $T=X_{1}+i X_{2}$ on $\mathcal{F}(\mathcal{H})$ is contained in $\left[-2\left\|v_{1}\right\|, 2\left\|v_{1}\right\|\right]+$ $i\left[-2\left\|v_{2}\right\|, 2\left\|v_{2}\right\|\right]$. Throughout this paper, we are interested in non-normal operators $T$; so we assume that $\operatorname{Im}\left\langle v_{2}, v_{1}\right\rangle$ is non-zero.

Lemma 2.1. If $A$ and $B$ are self-adjoint with $\sigma(A) \subseteq\left[r_{1}, r_{2}\right]$ and $\sigma(B) \subseteq\left[t_{1}, t_{2}\right]$, then $\sigma(A+i B) \subseteq$ $\left[r_{1}, r_{2}\right]+i\left[t_{1}, t_{2}\right]$.

Proof. If $A_{1}=A_{1}^{*}, B_{1} \geq 0$, and $B_{1}$ is invertible, then $A_{1}+i B_{1}=B_{1}^{\frac{1}{2}}\left(B_{1}^{-\frac{1}{2}} A_{1} B_{1}^{-\frac{1}{2}}+i\right) B_{1}^{\frac{1}{2}}$ is invertible since $B_{1}^{-\frac{1}{2}} A_{1} B_{1}^{-\frac{1}{2}}$ is self-adjoint. Suppose $a+i b \notin\left[r_{1}, r_{2}\right]+i\left[t_{1}, t_{2}\right]$. Then either $a<r_{1}$ or $a>r_{2}$ or $b<t_{1}$ or $b>t_{2}$. If $b<t_{1}$, then $A+i B-(a+i b)=(A-a)+i(B-b)$ and $B-b \geq 0$ is invertible. So $a+i b \notin \sigma(A+i B)$. If $b>t_{2}$, then $a+i b-(A+i B)=(a-A)+i(b-B)$ and $b-B \geq 0$ is invertible, so that $a+i b \notin \sigma(A+i B)$. Since $(A-a)+i(B-b)=i((B-b)-i(A-a))$, we can easily show that $A+i B-(a+i b)$ is invertible for each case of $a<r_{1}$ and $a>r_{2}$. Therefore, $\sigma(A+i B) \subseteq\left[r_{1}, r_{2}\right]+i\left[t_{1}, t_{2}\right]$.

The operator $T \in B(\mathcal{H})$ is said to be hyponormal, if its self-commutator $T^{*} T-T T^{*}$ is positive. Furthermore, if there is no reducing subspace of $T$, the restriction of $T$ to which is normal, then $T$ is said to be pure hyponormal or completely non-normal hyponormal.

Theorem 2.2 (Theorem 2.1.3 of $[6]$ ). Let $T \in B(\mathcal{H})$ be a hyponormal operator with $\left[T^{*}, T\right]=D$. Then there is a unique orthogonal decomposition $\mathcal{H}=\mathcal{H}_{p}(T) \oplus \mathcal{H}_{n}(T)$ where $\mathcal{H}_{p}(T)$ and $\mathcal{H}_{n}(T)$ are reducing subspaces for $T$, such that 
(i) $T_{p}=\left.T\right|_{\mathcal{H}_{p}(T)}$ is pure hyponormal,

(ii) $T_{n}=\left.T\right|_{\mathcal{H}_{n}(T)}$ is normal.

Moreover,

$$
\mathcal{H}_{p}(T)=\bigvee\left\{T^{* k} T^{l} D(\mathcal{H}) \mid k, l \in \mathbb{N}\right\} \quad \text { and } \quad \mathcal{H}_{n}(T)=\left\{\zeta \in \mathcal{H} \mid D T^{* l} T^{k} \zeta=0 \text { for every } k, l \in \mathbb{N}\right\} .
$$

As we can see in (3), if $\operatorname{Im}\left\langle v_{2}, v_{1}\right\rangle \leq 0$ (or $\geq 0$ ), then $T=X_{1}+i X_{2}$ is a hyponormal operator (or cohyponormal, respectively) on $\mathcal{F}(\mathcal{H})$. By Theorem 2.2 , the pure parts $\mathcal{H}_{p}(T)$ and $\mathcal{H}_{p}\left(T^{*}\right)$ of $T$ and $T^{*}$ are equal to $\overline{\operatorname{alg}\left(T, T^{*}, 1\right) \Omega}$.

Assuming that $\operatorname{Im}\left\langle v_{2}, v_{1}\right\rangle \leq 0$, if $v_{2}$ is a scalar mutiple of $v_{1}$, then $\operatorname{alg}\left(T, T^{*}, 1\right) \Omega$ is dense in $\mathcal{F}\left(\mathbb{C}\left\langle v_{1}\right\rangle\right)$ so that $T$ is pure hyponormal on $\mathcal{F}\left(\mathbb{C}\left\langle v_{1}\right\rangle\right)$. However, if $v_{2}$ is not a scalar multiple of $v_{1}$, then $T$ is not a pure hyponormal operator on $\mathcal{F}\left(\mathbb{C}\left\langle v_{1}, v_{2}\right\rangle\right)$, that is, there exists a nontrivial reducing subspace $\mathcal{N}$ of $T$ in $\mathcal{F}\left(\mathbb{C}\left\langle v_{1}, v_{2}\right\rangle\right)$ such that $\left.T\right|_{\mathcal{N}}$ is normal. For, suppose that $u$ is a unit vector which is orthogonal to $v_{1}$ in $\mathbb{C}\left\langle v_{1}, v_{2}\right\rangle$ and $v_{2}=c v_{1}+d u$ where $c, d \in \mathbb{C}$ are non-zero. Since $v_{2}$ and $\frac{c}{|c|^{2}} v_{1}-\frac{d}{|d|^{2}} u$ are orthogonal to each other, for each $m, n \in \mathbb{N}$,

$$
\left(l\left(v_{1}\right)+l\left(v_{1}\right)^{*}\right)^{m}\left(u \otimes\left(\frac{c}{|c|^{2}} v_{1}-\frac{d}{|d|^{2}} u\right)\right) \in \operatorname{span}\left\{v_{1}^{\otimes k} \otimes u \otimes\left(\frac{c}{|c|^{2}} v_{1}-\frac{d}{|d|^{2}} u\right) \mid k \in \mathbb{N}\right\}
$$

and

$$
\left(r\left(v_{2}\right)+r\left(v_{2}\right)^{*}\right)^{n}\left(u \otimes\left(\frac{c}{|c|^{2}} v_{1}-\frac{d}{|d|^{2}} u\right)\right) \in \operatorname{span}\left\{u \otimes\left(\frac{c}{|c|^{2}} v_{1}-\frac{d}{|d|^{2}} u\right) \otimes v_{2}^{\otimes k} \mid k \in \mathbb{N}\right\} .
$$

Since $\operatorname{alg}\left(T, T^{*}, 1\right)=\operatorname{alg}\left(X_{1}, X_{2}, 1\right)$ and $\left[X_{1}, X_{2}\right]=2 i\left(\operatorname{Im}\left\langle v_{2}, v_{1}\right\rangle\right) P$,

$$
\begin{aligned}
\mathcal{N} & :=\bigvee\left\{\left(r\left(v_{2}\right)+r\left(v_{2}\right)^{*}\right)^{n}\left(l\left(v_{1}\right)+l\left(v_{1}\right)^{*}\right)^{m}\left(u \otimes\left(\frac{c}{|c|^{2}} v_{1}-\frac{d}{|d|^{2}} u\right)\right) \mid m, n \in \mathbb{N}\right\} \\
& =\bigvee\left\{v_{1}^{\otimes m} \otimes u \otimes\left(\frac{c}{|c|^{2}} v_{1}-\frac{d}{|d|^{2}} u\right) \otimes v_{2}^{\otimes n} \mid m, n \in \mathbb{N}\right\} .
\end{aligned}
$$

is a nontrivial reducing subspace of $T$ in $\mathcal{F}\left(\mathbb{C}\left\langle v_{1}, v_{2}\right\rangle\right)$ which is orthogonal to $\mathbb{C} \Omega$. Clearly, the restrictions of $l\left(v_{1}\right)+l\left(v_{1}\right)^{*}$ and $r\left(v_{2}\right)+r\left(v_{2}\right)^{*}$ to $\mathcal{N}$ commute, so the restriction of $T$ to $\mathcal{N}$ is normal.

Now we will characterize the pure part $\overline{\operatorname{alg}\left(T, T^{*}, 1\right) \Omega}$ of $T$ in $\mathcal{F}(\mathcal{H})$ when $v_{1}$ and $v_{2}$ are linearly independent.

Proposition 2.3. Let $T=l\left(v_{1}\right)+l\left(v_{1}\right)^{*}+i\left(r\left(v_{2}\right)+r\left(v_{2}\right)^{*}\right)$ where $\left\|v_{1}\right\|=1$. Suppose $v_{2}=c v_{1}+d u$ where $c, d \in \mathbb{C}$ are non-zero, $u \perp v_{1}$ and $\|u\|=1$, and let $w:=\frac{1}{\sqrt{2}}\left(\frac{c}{|c|^{2}} v_{1}-\frac{d}{|d|^{2}} u\right)$. Let $A_{n}$ be the span of length $n$ tensor products in $\mathcal{F}\left(\mathbb{C}\left\langle v_{1}, v_{2}\right\rangle\right)$ for each $n \in \mathbb{N}$ and let $A_{0}=\mathbb{C} \Omega$. Then

$$
\overline{\operatorname{alg}\left(T, T^{*}, 1\right) \Omega}=\bigoplus_{n \geq 0}\left(A_{n} \cap \operatorname{alg}\left(T, T^{*}, 1\right) \Omega\right)
$$

and for every $n \in \mathbb{N}$,

$$
B_{n}:=\left\{v_{1}^{\otimes n}, v_{1}^{\otimes n-1} \otimes u, v_{1}^{\otimes n-2} \otimes u \otimes v_{2}, \cdots, v_{1} \otimes u \otimes v_{2}^{\otimes n-2}, u \otimes v_{2}^{\otimes n-1}\right\}
$$

and

$$
B_{n}^{\prime}:=\left\{v_{2}^{\otimes n}, w \otimes v_{2}^{\otimes n-1}, v_{1} \otimes w \otimes v_{2}^{\otimes n-2}, \cdots, v_{1}^{\otimes n-2} \otimes w \otimes v_{2}, v_{1}^{\otimes n-1} \otimes w\right\}
$$

are orthogonal bases of $A_{n} \cap \operatorname{alg}\left(T, T^{*}, 1\right) \Omega$. Furthermore, we have the obvious isomorphisms

$$
\begin{aligned}
\overline{\operatorname{alg}\left(T, T^{*}, 1\right) \Omega} & \cong \mathcal{F}\left(\mathbb{C}\left\langle v_{1}\right\rangle\right) \oplus\left(\mathcal{F}\left(\mathbb{C}\left\langle v_{1}\right\rangle\right) \otimes u \otimes \mathcal{F}\left(\mathbb{C}\left\langle v_{2}\right\rangle\right)\right) \\
& \cong \mathcal{F}\left(\mathbb{C}\left\langle v_{2}\right\rangle\right) \oplus\left(\mathcal{F}\left(\mathbb{C}\left\langle v_{1}\right\rangle\right) \otimes w \otimes \mathcal{F}\left(\mathbb{C}\left\langle v_{2}\right\rangle\right)\right) .
\end{aligned}
$$

Proof. We will prove by induction on $n$ that $B_{n}$ is an orthogonal basis for $A_{n} \cap \operatorname{alg}\left(T, T^{*}, 1\right)$. This is clear for $n=1$. For $n=2$, consider the orthogonal basis of $A_{2}$

$$
Z_{2}=\left\{v_{1}^{\otimes 2}, v_{1} \otimes u, u \otimes v_{2}, u \otimes w\right\}
$$


containing $B_{2}$. Here, $B_{2}=\left\{v_{1}^{\otimes 2}, v_{1} \otimes u, u \otimes v_{2}\right\} \subseteq \operatorname{alg}\left(T, T^{*}, 1\right) \Omega$ and $Z_{2} \backslash B_{2}=\{u \otimes w\} \subseteq\left(\operatorname{alg}\left(T, T^{*}, 1\right) \Omega\right)^{\perp}$ as we saw in the above argument describing $\mathcal{N}$. Now the assertion is proved for $n=2$.

Consider another orthogonal basis of $A_{2}, Z_{2}^{\prime}=\left\{v_{2}^{\otimes 2}, v_{2} \otimes w, w \otimes v_{2}, w^{\otimes 2}\right\}$. Then $Z_{3}:=\left\{v_{1} \otimes Z_{2}\right\} \cup\{u \otimes$ $\left.Z_{2}^{\prime}\right\}$ is an orthogonal basis of $A_{3}$. Since $\operatorname{alg}\left(T, T^{*}, 1\right) \Omega$ is a reducing subspace of $T, v_{1} \otimes B_{2} \subseteq \operatorname{alg}\left(T, T^{*}, 1\right) \Omega$ and $v_{1} \otimes\left\{Z_{2} \backslash B_{2}\right\} \subseteq\left(\operatorname{alg}\left(T, T^{*}, 1\right) \Omega\right)^{\perp}$. In $u \otimes Z_{2}^{\prime}$, only $u \otimes v_{2}^{\otimes 2}$ is contained in $\operatorname{alg}\left(T, T^{*}, 1\right) \Omega$ because every tensor product in $\mathcal{F}\left(\mathbb{C}\left\langle v_{1}, v_{2}\right\rangle\right)$ which starts with $u$ and ends with $w$ belongs to $\mathcal{N}$ and is therefore orthogonal to $\operatorname{alg}\left(T, T^{*}, 1\right) \Omega$; moreover $u \otimes w \otimes v_{2}=\left(r\left(v_{2}\right)+r\left(v_{2}\right)^{*}\right)(u \otimes w) \in\left(\operatorname{alg}\left(T, T^{*}, 1\right) \Omega\right)^{\perp}$. Hence, $B_{3}=\left\{v_{1} \otimes B_{2}\right\} \cup\left\{u \otimes v_{2}^{\otimes 2}\right\}$ is contained in $\operatorname{alg}\left(T, T^{*}, 1\right) \Omega$ and $Z_{3} \backslash B_{3}$ is contained in $\left(\operatorname{alg}\left(T, T^{*}, 1\right) \Omega\right)^{\perp}$. Thus the assertion holds for $n=3$.

The induction step for general $n$ proceeds similarly. For each $n \in \mathbb{N}$, construct an orthogonal basis $Z_{n}$ for $A_{n}$ as follows.

$$
Z_{n}=\left\{v_{1}^{n}\right\} \cup\left(\bigcup_{1 \leq j \leq n}\left\{v_{1}^{n-j} \otimes u \otimes Z_{j-1}^{\prime}\right\}\right),
$$

where $Z_{k}^{\prime}$ is the set of all length $k$ tensor products in $\mathcal{F}(\mathcal{H})$ whose components consist of $v_{2}$ and $w$. The induction hypothesis is that $B_{j}$ is an orthogonal basis of $A_{j} \cap \operatorname{alg}\left(T, T^{*}, 1\right) \Omega$ and $Z_{j} \backslash B_{j}$ is orthogonal to $\operatorname{alg}\left(T, T^{*}, 1\right) \Omega$ for each $1 \leq j \leq n$. Then $Z_{n+1}=\left\{v_{1} \otimes Z_{n}\right\} \cup\left\{u \otimes Z_{n}^{\prime}\right\}$ and it is an orthogonal basis of $A_{n+1}$. Since $\operatorname{alg}\left(T, T^{*}, 1\right) \Omega$ is a reducing subspace of $T$ and is invariant under $l\left(v_{1}\right)+l\left(v_{1}\right)^{*}$, we have $v_{1} \otimes B_{n}=\left\{v_{1}^{\otimes n+1}, v_{1}^{\otimes n} \otimes u, v_{1}^{\otimes n-1} \otimes u \otimes v_{2}, \cdots, v_{1} \otimes u \otimes v_{2}^{\otimes n-1}\right\} \subseteq \operatorname{alg}\left(T, T^{*}, 1\right) \Omega$ and $v_{1} \otimes\left\{Z_{n} \backslash B_{n}\right\} \subseteq$ $\left(\operatorname{alg}\left(T, T^{*}, 1\right) \Omega\right)^{\perp}$. In $u \otimes Z_{n}^{\prime}$, only $u \otimes v_{2}^{\otimes n}$ is contained in $\operatorname{alg}\left(T, T^{*}, 1\right) \Omega$ and the other elements are orthogonal to $\operatorname{alg}\left(T, T^{*}, 1\right) \Omega$ by the induction hypothesis. Therefore, $B_{n+1}=\left\{v_{1} \otimes B_{n}\right\} \cup\left\{u \otimes v_{2}^{\otimes n}\right\}$ is an orthogonal basis for $A_{n+1} \cap \operatorname{alg}\left(T, T^{*}, 1\right) \Omega$ and $Z_{n+1} \backslash B_{n+1}$ is an orthogonal basis for $A_{n+1} \cap$ $\left(\operatorname{alg}\left(T, T^{*}, 1\right) \Omega\right)^{\perp}$. Thus, for every $n \in \mathbb{N}, B_{n}$ is an orthogonal basis for the set of all length $n$ tensor products in $\operatorname{alg}\left(T, T^{*}, 1\right) \Omega$. This finishes the proof by induction.

The proof that for $n \in \mathbb{N}, B_{n}^{\prime}$ is also an orthogonal basis for $A_{n} \cap \operatorname{alg}\left(T, T^{*}, 1\right) \Omega$ follows similarly by induction on $n$, using the invariance of $\operatorname{alg}\left(T, T^{*}, 1\right) \Omega$ under $r\left(v_{2}\right)+r\left(v_{2}\right)^{*}$ rather than $l\left(v_{1}\right)+l\left(v_{1}\right)^{*}$.

The equality (4) follows by the above proofs.

Before we further investigate the operator $T=X_{1}+i X_{2}$ having $v_{1}$ and $v_{2}$ linearly independent, we will take a look at the case when the vectors $v_{1}$ and $v_{2}$ are linearly dependent. We will refer to the following result.

Theorem 2.4 ([2]). If $T$ is a hyponormal operator on $\mathcal{H}$, then $C^{*}(T)$ is generated by the unilateral shift if and only if $T$ is unitarily equivalent to $S$, where $S$ satisfies conditions

(i) $S$ is irreducible;

(ii) self-commutator $S^{*} S-S S^{*}$ is compact;

(iii) $\sigma_{e}(S)$ is a simple closed curve;

(iv) $\sigma(S)$ is the closure of $V$, where $V$ is the bounded component of $\mathbb{C} \backslash \sigma_{e}(S)$;

(v) for $\lambda \in \sigma(S) \backslash \sigma_{e}(S)$, ind $(S-\lambda)=-1$.

Example 2.5. Let $v_{1}=\alpha v_{2}, \alpha \in \mathbb{C}, \operatorname{Im} \alpha \neq 0$, and $\left\|v_{2}\right\|=1$. Let $T$ be given by

$$
\begin{aligned}
T & =l\left(v_{1}\right)+l\left(v_{1}\right)^{*}+i\left(r\left(v_{2}\right)+r\left(v_{2}\right)^{*}\right) \\
& =\left(\alpha l\left(v_{2}\right)+i r\left(v_{2}\right)\right)+\left(\bar{\alpha} l\left(v_{2}\right)^{*}+i r\left(v_{2}\right)^{*}\right)
\end{aligned}
$$

on $\mathcal{F}(\mathbb{C})$. Then,

and for each $n \in \mathbb{N}$,

$$
T(\Omega)=(\alpha+i) v_{2}
$$

$T\left(v_{2}^{\otimes n}\right)=(\alpha+i) v_{2}^{\otimes n+1}+(\bar{\alpha}+i) v_{2}^{\otimes n-1}$.

Therefore,

$$
T=(\alpha+i) U+(\bar{\alpha}+i) U^{*}
$$

where $U$ is the unilateral shift on $\mathcal{F}(\mathbb{C})$.

If $\alpha=i$, then $T=2 i U$ and $\left[T^{*}, T\right]=4 P$ so that $T$ is a hyponormal operator. If $\alpha=-i$, then $T=2 i U^{*}$ and $\left[T^{*}, T\right]=-4 P$, so $T$ is cohyponormal. 
Since the image of the unilateral shift $U$ in the Calkin algebra is a normal operator, by the functional calculus, we have

$$
\begin{aligned}
\sigma_{e}\left((\alpha+i) U+(\bar{\alpha}+i) U^{*}\right) & =\left\{(\alpha+i) t+(\bar{\alpha}+i) \bar{t} \mid t \in \sigma_{e}(U)\right\} \\
& =\{\alpha t+\overline{\alpha t}+i(t+\bar{t}) \mid t \in \mathbb{T}\}
\end{aligned}
$$

This curve is the solution set of

$$
x^{2}+|\alpha|^{2} y^{2}-2(\operatorname{Re} \alpha) x y=4(\operatorname{Im} \alpha)^{2}
$$

in the $x y$-plane, which is an ellipse centered at the origin. So the essential spectrum of $T$ is a simple closed curve. Let $V_{0}$ be the bounded component of $\mathbb{C} \backslash \sigma_{e}(T)$. Then by Theorem 2.4 we have

$$
\sigma(T)=\overline{V_{0}},
$$

and for $\lambda \in \sigma(T) \backslash \sigma_{e}(T)$,

$$
\operatorname{ind}(T-\lambda)= \begin{cases}-1, & \operatorname{Im}(\alpha)>0 \\ 1, & \operatorname{Im}(\alpha)<0 .\end{cases}
$$

Thus, the principal function is the characteristic function of the interior of the ellipse 77 when $\operatorname{Im} \alpha<0$, and is the negative of this when $\operatorname{Im} \alpha>0$.

2.2. In the rest of this paper, we consider the pure part of $T=X_{1}+i X_{2}$ acting on $\overline{\operatorname{alg}\left(T, T^{*}, 1\right) \Omega}$ where $X_{1}=l\left(v_{1}\right)+l\left(v_{1}\right)^{*}$ and $X_{2}=r\left(v_{2}\right)+r\left(v_{2}\right)^{*}$. So $T$ is a completely non-normal operator.

Now we will find a formula for the principal function of $T$ when $v_{1}$ and $v_{2}$ are linearly independent. For this, we will use equation (2); so we will first establish a formula for $\operatorname{det} E(l, s)$ of $T$. Suppose $l \in \mathbb{C} \backslash \sigma\left(X_{2}\right)$ and $s \in \mathbb{C} \backslash \sigma\left(X_{1}\right)$. From (1), we have

$$
\begin{aligned}
\operatorname{det} E(l, s) & =\operatorname{det}\left(\left(X_{2}-l\right)\left(X_{1}-s\right)\left(X_{2}-l\right)^{-1}\left(X_{1}-s\right)^{-1}\right) \\
& =\operatorname{det}\left(\left(\left(X_{1}-s\right)\left(X_{2}-l\right)-2 \operatorname{Im}\left\langle v_{2}, v_{1}\right\rangle i P\right)\left(X_{2}-l\right)^{-1}\left(X_{1}-s\right)^{-1}\right) \\
& =\operatorname{det}\left(1-2 \operatorname{Im}\left\langle v_{2}, v_{1}\right\rangle i P\left(X_{2}-l\right)^{-1}\left(X_{1}-s\right)^{-1}\right) \\
& =\operatorname{det}\left(1-2 \operatorname{Im}\left\langle v_{2}, v_{1}\right\rangle i P^{2}\left(X_{2}-l\right)^{-1}\left(X_{1}-s\right)^{-1}\right) \\
& =\operatorname{det}\left(1-2 \operatorname{Im}\left\langle v_{2}, v_{1}\right\rangle i P\left(X_{2}-l\right)^{-1}\left(X_{1}-s\right)^{-1} P\right) \\
& =\operatorname{det}\left(1-2 \operatorname{Im}\left\langle v_{2}, v_{1}\right\rangle i \varphi_{\Omega}\left(\left(X_{2}-l\right)^{-1}\left(X_{1}-s\right)^{-1}\right) P\right) \\
& =1-2 \operatorname{Im}\left\langle v_{2}, v_{1}\right\rangle i \varphi_{\Omega}\left(\left(X_{2}-l\right)^{-1}\left(X_{1}-s\right)^{-1}\right) \\
& =1-2 \operatorname{Im}\left\langle v_{2}, v_{1}\right\rangle i \overline{\varphi_{\Omega}\left(\left(\bar{s}-X_{1}\right)^{-1}\left(\bar{l}-X_{2}\right)^{-1}\right)} \\
& =1-2 \operatorname{Im}\left\langle v_{2}, v_{1}\right\rangle i \overline{G_{\left(X_{1}, X_{2}\right)}(\bar{s}, \bar{l})}
\end{aligned}
$$

where $G_{\left(X_{1}, X_{2}\right)}(z, w)=\varphi\left(\left(z-X_{1}\right)^{-1}\left(w-X_{2}\right)^{-1}\right)$. Note that $G_{\left(X_{1}, X_{2}\right)}(z, w)$ is the germ of a holomorphic function near $(\infty, \infty)$ in $\mathbb{C}_{\infty} \times \mathbb{C}_{\infty}$ (see [8]).

2.3. We review the definition and formula of the partial bi-free R-transform, $R_{(a, b)}(z, w)$, defined in 88 and find $\operatorname{det} E(l, s)$ in terms of $l$ and $s$.

Definition 2.6 $([8])$. Let $(a, b)$ be a two-faced pair of non-commutative random variables in $(\mathcal{A}, \varphi)$. Set $I=\{i\}$ and $J=\{j\}$ and suppose $\alpha:\{1, \cdots, m+n\} \rightarrow I \amalg J$ is given by $\alpha(k)=i$ if $1 \leq k \leq m$ and $\alpha(k)=j$ if $m+1 \leq k \leq m+n$. We shall denote the bi-free cummulant $R_{\alpha}$ as $R_{m, n}$. The partial bi-free $R$-transform is the generating series

$$
R_{(a, b)}(z, w)=\sum_{\substack{m \geq 0, n \geq 0 \\ m+n \geq 1}} R_{m, n}(a, b) z^{m} w^{n} .
$$


Theorem 2.7 (Theorem 2.4 of $[8]$ ). We have the equality of germs of holomorphic functions near $(0,0) \in$ $\mathbb{C}^{2}$,

$$
R_{(a, b)}(z, w)=1+z R_{a}(z)+w R_{b}(w)-\frac{z w}{G_{(a, b)}\left(K_{a}(z), K_{b}(w)\right)},
$$

where $R_{a}(z)$ and $R_{b}(w)$ are one variable $R$-transforms, and $K_{a}(z)=z^{-1}+R_{a}(z)$ and $K_{b}(w)=w^{-1}+$ $R_{b}(w)$.

For the given two-faced pair $\left(X_{1}, X_{2}\right)$, the definition of the partial bi-free R-transform and Lemma 7.2 of 7 give

$$
\begin{aligned}
R_{\left(X_{1}, X_{2}\right)}(z, w) & =R_{2,0}\left(X_{1}, X_{2}\right)+R_{0,2}\left(X_{1}, X_{2}\right)+R_{1,1}\left(X_{1}, X_{2}\right) \\
& =\varphi\left(X_{1}^{2}\right) z^{2}+\varphi\left(X_{2}^{2}\right) w^{2}+\varphi\left(X_{1} X_{2}\right) z w \\
& =\left\|v_{1}\right\|^{2} z^{2}+\left\|v_{2}\right\|^{2} w^{2}+\left\langle v_{2}, v_{1}\right\rangle z w .
\end{aligned}
$$

From the formula for the partial bi-free R-transform in Theorem 2.7, we also have

$$
R_{\left(X_{1}, X_{2}\right)}(z, w)=1+\left\|v_{1}\right\|^{2} z^{2}+\left\|v_{2}\right\|^{2} w^{2}-\frac{z w}{G_{\left(X_{1}, X_{2}\right)}\left(\frac{1}{z}+\left\|v_{1}\right\|^{2} z, \frac{1}{w}+\left\|v_{2}\right\|^{2} w\right)}
$$

Denoting

$$
t_{1}=\frac{1}{z}+\left\|v_{1}\right\|^{2} z \quad \text { and } \quad t_{2}=\frac{1}{w}+\left\|v_{2}\right\|^{2} w
$$

for $z, w \in \mathbb{C} \backslash\{0\}$ close to 0 , we have

$$
z=\frac{t_{1}-\sqrt{t_{1}^{2}-4\left\|v_{1}\right\|^{2}}}{2\left\|v_{1}\right\|^{2}} \quad \text { and } \quad w=\frac{t_{2}-\sqrt{t_{2}^{2}-4\left\|v_{2}\right\|^{2}}}{2\left\|v_{2}\right\|^{2}}
$$

where the branches of the square roots are $\sqrt{t_{1}^{2}-4\left\|v_{1}\right\|^{2}} \approx t_{1}$ and $\sqrt{t_{2}^{2}-4\left\|v_{2}\right\|^{2}} \approx t_{2}$ for $\left|t_{1}\right|$ and $\left|t_{2}\right|$ large. From the formulas $(9)$ and $(10)$, we get

$$
\begin{aligned}
G_{\left(X_{1}, X_{2}\right)}\left(t_{1}, t_{2}\right) & =\frac{z w}{1-\left\langle v_{2}, v_{1}\right\rangle z w} \\
& =\frac{\left(t_{1}-\sqrt{t_{1}^{2}-4\left\|v_{1}\right\|^{2}}\right)\left(t_{2}-\sqrt{t_{2}^{2}-4\left\|v_{2}\right\|^{2}}\right)}{4\left\|v_{1}\right\|^{2}\left\|v_{2}\right\|^{2}-\left\langle v_{2}, v_{1}\right\rangle\left(t_{1}-\sqrt{t_{1}^{2}-4\left\|v_{1}\right\|^{2}}\right)\left(t_{2}-\sqrt{t_{2}^{2}-4\left\|v_{2}\right\|^{2}}\right)},
\end{aligned}
$$

when $\left|t_{1}\right|$ and $\left|t_{2}\right|$ are large.

Let

$$
q(t)=\frac{t-\sqrt{t^{2}-4}}{2} \quad(t \in \mathbb{C} \backslash[-2,2])
$$

The function $z \mapsto z+\frac{1}{z}$ sends the punctured unit disk $\{z|0<| z \mid<1\}$ biholomorphically onto $\mathbb{C} \backslash[-2,2]$. The function $q$ is its inverse with respect to composition. We deduce that the identity $q(t)=\overline{q(\bar{t})}$ holds for all $t \in \mathbb{C} \backslash[-2,2]$.

By (8) and (11), for $|l|$ and $|s|$ large, we have

$$
\begin{aligned}
\operatorname{det} E(l, s) & =1-2\left(\operatorname{Im}\left\langle v_{2}, v_{1}\right\rangle\right) i\left(\frac{\left(\bar{s}-\sqrt{\bar{s}^{2}-4\left\|v_{1}\right\|^{2}}\right)\left(\bar{l}-\sqrt{\bar{l}^{2}-4\left\|v_{2}\right\|^{2}}\right)}{4\left\|v_{1}\right\|^{2}\left\|v_{2}\right\|^{2}-\left\langle v_{2}, v_{1}\right\rangle\left(\bar{s}-\sqrt{\bar{s}^{2}-4\left\|v_{1}\right\|^{2}}\right)\left(\bar{l}-\sqrt{\bar{l}^{2}-4\left\|v_{2}\right\|^{2}}\right)}\right) \\
& \left.=1+\frac{2\left(\operatorname{Im}\left\langle v_{1}, v_{2}\right\rangle\right) i\left(s-\sqrt{s^{2}-4\left\|v_{1}\right\|^{2}}\right)\left(l-\sqrt{l^{2}-4\left\|v_{2}\right\|^{2}}\right)}{4\left\|v_{1}\right\|^{2}\left\|v_{2}\right\|^{2}-\left\langle v_{1}, v_{2}\right\rangle\left(s-\sqrt{s^{2}-4\left\|v_{1}\right\|^{2}}\right)\left(l-\sqrt{l^{2}-4\left\|v_{2}\right\|^{2}}\right.}\right)
\end{aligned}
$$




$$
\begin{aligned}
& =\frac{4\left\|v_{1}\right\|^{2}\left\|v_{2}\right\|^{2}-\overline{\left\langle v_{1}, v_{2}\right\rangle}\left(s-\sqrt{s^{2}-4\left\|v_{1}\right\|^{2}}\right)\left(l-\sqrt{l^{2}-4\left\|v_{2}\right\|^{2}}\right)}{4\left\|v_{1}\right\|^{2}\left\|v_{2}\right\|^{2}-\left\langle v_{1}, v_{2}\right\rangle\left(s-\sqrt{s^{2}-4\left\|v_{1}\right\|^{2}}\right)\left(l-\sqrt{l^{2}-4\left\|v_{2}\right\|^{2}}\right)} \\
& =\frac{1-\frac{\bar{\alpha}}{\left\|v_{1}\right\|\left\|v_{2}\right\|} q\left(\frac{s}{\left\|v_{1}\right\|}\right) q\left(\frac{l}{\left\|v_{2}\right\|}\right)}{1-\frac{\alpha}{\left\|v_{1}\right\|\left\|v_{2}\right\|} q\left(\frac{s}{\left\|v_{1}\right\|}\right) q\left(\frac{l}{\left\|v_{2}\right\|}\right)},
\end{aligned}
$$

where $\alpha=\left\langle v_{1}, v_{2}\right\rangle$, and for the second equality, we have used

$$
\overline{\left(\bar{s}-\sqrt{\bar{s}^{2}-4\left\|v_{1}\right\|^{2}}\right)}=2\left\|v_{1}\right\| \overline{\left(\frac{\bar{s}}{\left\|v_{1}\right\|}\right)}=2\left\|v_{1}\right\| q\left(\frac{s}{\left\|v_{1}\right\|}\right)=s-\sqrt{s^{2}-4\left\|v_{1}\right\|^{2}}
$$

and

$$
\overline{\left(\bar{l}-\sqrt{\bar{l}^{2}-4\left\|v_{2}\right\|^{2}}\right)}=2\left\|v_{2}\right\| \overline{\left(\frac{\bar{l}}{\left\|v_{2}\right\|}\right)}=2\left\|v_{2}\right\| q\left(\frac{l}{\left\|v_{2}\right\|}\right)=l-\sqrt{l^{2}-4\left\|v_{2}\right\|^{2}} .
$$

Since $v_{1}$ and $v_{2}$ are linearly independent, $|\alpha|<\left\|v_{1}\right\|\left\|v_{2}\right\|$. Since $\left|q\left(\frac{s}{\left\|v_{1}\right\|}\right)\right|<1$ and $\left|q\left(\frac{l}{\left\|v_{2}\right\|}\right)\right|<1$ for $s \in \mathbb{C} \backslash\left[-2\left\|v_{1}\right\|, 2\left\|v_{1}\right\|\right]$ and $l \in \mathbb{C} \backslash\left[-2\left\|v_{2}\right\|, 2\left\|v_{2}\right\|\right]$, the numerator and denominator in (13) do not vanish for such $s$ and $l$. So the right-hand side of $(13)$ is a holomorphic function there. Since by definition in (8), $\operatorname{det} E(l, s)$ is holomorphic on $\left(\mathbb{C}_{\infty} \backslash \sigma\left(X_{2}\right)\right) \times\left(\mathbb{C}_{\infty} \backslash \sigma\left(X_{1}\right)\right)$, it follows from the analytic continuation that the formula of $\operatorname{det} E(l, s)$ in $(13)$ holds for all $s \in \mathbb{C} \backslash\left[-2\left\|v_{1}\right\|, 2\left\|v_{1}\right\|\right]$ and $l \in \mathbb{C} \backslash\left[-2\left\|v_{2}\right\|, 2\left\|v_{2}\right\|\right]$.

2.4. In this subsection, we find the formula of the principal function $g(\delta, \gamma)$ of $T$ by using the formula 13). The principal function $g$ was defined on $\mathbb{R}^{2}$ by

$$
\operatorname{det} E(l, s)=\exp \left(\frac{1}{2 \pi i} \int_{\mathbb{R}} \int_{\mathbb{R}} g(\delta, \gamma) \frac{d \delta}{\delta-l} \frac{d \gamma}{\gamma-s}\right)
$$

and $\operatorname{supp}(g) \subseteq\left\{(\delta, \gamma) \in \mathbb{R}^{2} \mid \gamma+i \delta \in \sigma(T)\right\}$. To find the principal function of $T$, consider the function $f$ defined by

$$
f(l, \gamma)=\int_{\mathbb{R}} g(\delta, \gamma) \frac{d \delta}{\delta-l}
$$

for $l \in \mathbb{C} \backslash \sigma\left(X_{2}\right)$ and $\gamma \in \mathbb{R}$. Fixing $\gamma \in \mathbb{R}, f(l, \gamma)$ is a holomorphic function for $l \in \mathbb{C} \backslash \sigma\left(X_{2}\right)$. From (13) and the definition of $g(\delta, \gamma)$, we have

$$
\int_{\mathbb{R}} f(l, \gamma) \frac{d \gamma}{\gamma-s}=(2 \pi i) \log \left(\frac{1-\frac{\bar{\alpha}}{\left\|v_{1}\right\|\left\|v_{2}\right\|} q\left(\frac{s}{\left\|v_{1}\right\|}\right) q\left(\frac{l}{\left\|v_{2}\right\|}\right)}{1-\frac{\alpha}{\left\|v_{1}\right\|\left\|v_{2}\right\|} q\left(\frac{s}{\left\|v_{1}\right\|}\right) q\left(\frac{l}{\left\|v_{2}\right\|}\right)}\right),
$$

where $s \in \mathbb{C}_{\infty} \backslash \sigma\left(X_{1}\right)$ and $l \in \mathbb{C}_{\infty} \backslash \sigma\left(X_{2}\right)$. Now we will find the function $f(l, \gamma)$ by using the Stieltjes inversion formula.

We defined the function $q(t)$ for $t \in \mathbb{C} \backslash[-2,2]$ in 12 .

Lemma 2.8. If $t_{0} \in[-2,2]$, then

$$
\lim _{\epsilon \searrow 0} q\left(t_{0}+i \epsilon\right)=\frac{t_{0}-i \sqrt{4-t_{0}^{2}}}{2}
$$

Proof. For $t_{0} \in(-2,2)$,

$$
\begin{aligned}
\lim _{\epsilon \searrow 0} q\left(t_{0}+i \epsilon\right) & =\lim _{\epsilon \searrow 0} \frac{t_{0}+i \epsilon-\sqrt{\left(t_{0}+i \epsilon\right)^{2}-4}}{2} \\
& =\lim _{\epsilon \searrow 0} \frac{t_{0}+i \epsilon-\sqrt{-\left(4+\epsilon^{2}-t_{0}^{2}\right)+2 i \epsilon t_{0}}}{2} \\
& =\frac{t_{0}-i \sqrt{4-t_{0}^{2}}}{2} .
\end{aligned}
$$


For, when $\epsilon$ is large and positive, the branch of a square root is such that $\sqrt{-\left(4+\epsilon^{2}-t_{0}^{2}\right)+2 i \epsilon t_{0}} \approx t_{0}+i \epsilon$. So $\lim _{\epsilon \searrow 0} \sqrt{-\left(4+\epsilon^{2}-t_{0}^{2}\right)+2 i \epsilon t_{0}}=i \sqrt{4-t_{0}^{2}}$.

Define a function $\zeta(t)$ for $t \in[-2,2]$ by

$$
\zeta(t)=\frac{t-i \sqrt{4-t^{2}}}{2}
$$

Then $\zeta(t) \in \mathbb{T}$ for $t \in[-2,2]$, where $\mathbb{T}$ is a unit circle in $\mathbb{C}$. By Lemma 2.8, the limit of $q(t+i \epsilon)$ goes to $\zeta(t)$ as $\epsilon \searrow 0$, where $t \in[-2,2]$. Then we have for $\gamma \in\left[-2\left\|v_{1}\right\|, 2\left\|v_{1}\right\|\right]$,

$$
\lim _{\epsilon \searrow 0} q\left(\frac{\gamma}{\left\|v_{1}\right\|}+i \frac{\epsilon}{\left\|v_{1}\right\|}\right)=\frac{\frac{\gamma}{\left\|v_{1}\right\|}-i \sqrt{4-\left(\frac{\gamma}{\left\|v_{1}\right\|}\right)^{2}}}{2}=\zeta\left(\frac{\gamma}{\left\|v_{1}\right\|}\right) \in \mathbb{T} .
$$

Fix $l \in \mathbb{R} \backslash\left[-2\left\|v_{2}\right\|, 2\left\|v_{2}\right\|\right]$. Since clearly $f(l, \gamma)=0$ for $\gamma \in \mathbb{R} \backslash \sigma\left(X_{1}\right)$, we suppose $\gamma \in \sigma\left(X_{1}\right)$. Using (14), the Stieltjes inversion formula, and (15), we have

$$
\begin{aligned}
f(l, \gamma)= & \frac{1}{\pi} \lim _{\epsilon \searrow 0} \operatorname{Im}\left((2 \pi i) \log \left(\frac{1-\frac{\bar{\alpha}}{\left\|v_{1}\right\|\left\|v_{2}\right\|} q\left(\frac{\gamma}{\left\|v_{1}\right\|}+i \frac{\epsilon}{\left\|v_{1}\right\|}\right) q\left(\frac{l}{\left\|v_{2}\right\|}\right)}{\| \frac{\alpha}{\left\|v_{1}\right\|\left\|v_{2}\right\|} q\left(\frac{\gamma}{\left\|v_{1}\right\|}+i \frac{\epsilon}{\left\|v_{1}\right\|}\right) q\left(\frac{l}{\left\|v_{2}\right\|}\right)}\right)\right) \\
= & 2 \log \left|\frac{1-\frac{\bar{\alpha}}{\left\|v_{1}\right\|\left\|v_{2}\right\|} \zeta\left(\frac{\gamma}{\left\|v_{1}\right\|}\right) q\left(\frac{l}{\left\|v_{2}\right\|}\right)}{1-\frac{\alpha}{\left\|v_{1}\right\|\left\|v_{2}\right\|} \zeta\left(\frac{\gamma}{\left\|v_{1}\right\|}\right) q\left(\frac{l}{\left\|v_{2}\right\|}\right)}\right| \\
= & \log \frac{\left(1-\frac{\bar{\alpha}}{\left\|v_{1}\right\|\left\|v_{2}\right\|} \zeta\left(\frac{\gamma}{\left\|v_{1}\right\|}\right) q\left(\frac{l}{\left\|v_{2}\right\|}\right)\right)\left(1-\frac{\alpha}{\left\|v_{1}\right\|\left\|v_{2}\right\|} \zeta\left(\frac{\gamma}{\left\|v_{1}\right\|}\right) q\left(\frac{l}{\left\|v_{2}\right\|}\right)\right)}{\left(1-\frac{\alpha}{\left\|v_{1}\right\|\left\|v_{2}\right\|} \zeta\left(\frac{\gamma}{\left\|v_{1}\right\|}\right) q\left(\frac{l}{\left\|v_{2}\right\|}\right)\right)\left(1-\frac{\bar{\alpha}}{\left\|v_{1}\right\|\left\|v_{2}\right\|} \zeta\left(\frac{\gamma}{\left\|v_{1}\right\|}\right) q\left(\frac{l}{\left\|v_{2}\right\|}\right)\right)} \\
= & \log \left(1-\frac{\bar{\alpha}}{\left\|v_{1}\right\|\left\|v_{2}\right\|} \zeta\left(\frac{\gamma}{\left\|v_{1}\right\|}\right) q\left(\frac{l}{\left\|v_{2}\right\|}\right)\right)+\log \left(1-\frac{\alpha}{\left\|v_{1}\right\|\left\|v_{2}\right\|} \zeta\left(\frac{\gamma}{\left\|v_{1}\right\|}\right) q\left(\frac{l}{\left\|v_{2}\right\|}\right)\right) \\
& -\log \left(1-\frac{\alpha}{\left\|v_{1}\right\|\left\|v_{2}\right\|} \zeta\left(\frac{\gamma}{\left\|v_{1}\right\|}\right) q\left(\frac{l}{\left\|v_{2}\right\|}\right)\right)-\log \left(1-\frac{\bar{\alpha}}{\left\|v_{1}\right\|\left\|v_{2}\right\|} \zeta\left(\frac{\gamma}{\left\|v_{1}\right\|}\right) q\left(\frac{l}{\left\|v_{2}\right\|}\right)\right)
\end{aligned}
$$

where $\log$ is the principal branch of the logarithm. This equality holds where $\gamma \in \sigma\left(X_{1}\right)$ and $l \in$ $\mathbb{R} \backslash\left[-2\left\|v_{2}\right\|, 2\left\|v_{2}\right\|\right]$.

Fix $\gamma \in \sigma\left(X_{1}\right)$. Since each expression appearing as an argument of Log, above, remains in the disk of radius 1 centered at 1 for $l \in \mathbb{C} \backslash\left[-2\left\|v_{2}\right\|, 2\left\|v_{2}\right\|\right]$. So the expression (17) is holomorphic on $\mathbb{C} \backslash\left[-2\left\|v_{2}\right\|, 2\left\|v_{2}\right\|\right]$. The equality (16) was derived for $l \in \mathbb{R} \backslash\left[-2\left\|v_{2}\right\|, 2\left\|v_{2}\right\|\right]$, but as defined, $f(l, \gamma)$ is holomorphic in $\mathbb{C} \backslash\left[-2\left\|v_{2}\right\|, 2\left\|v_{2}\right\|\right]$. By analytic continuation, we have

$$
\begin{aligned}
\int_{\mathbb{R}} g(\delta, \gamma) \frac{d \delta}{\delta-l} & \\
= & \log \left(1-\frac{\bar{\alpha}}{\left\|v_{1}\right\|\left\|v_{2}\right\|} \zeta\left(\frac{\gamma}{\left\|v_{1}\right\|}\right) q\left(\frac{l}{\left\|v_{2}\right\|}\right)\right)+\log \left(1-\frac{\alpha}{\left\|v_{1}\right\|\left\|v_{2}\right\|} \overline{\zeta\left(\frac{\gamma}{\left\|v_{1}\right\|}\right)} q\left(\frac{l}{\left\|v_{2}\right\|}\right)\right) \\
& \quad-\log \left(1-\frac{\alpha}{\left\|v_{1}\right\|\left\|v_{2}\right\|} \zeta\left(\frac{\gamma}{\left\|v_{1}\right\|}\right) q\left(\frac{l}{\left\|v_{2}\right\|}\right)\right)-\log \left(1-\frac{\bar{\alpha}}{\left\|v_{1}\right\|\left\|v_{2}\right\|} \zeta\left(\frac{\gamma}{\left\|v_{1}\right\|}\right) q\left(\frac{l}{\left\|v_{2}\right\|}\right)\right)
\end{aligned}
$$

for $\gamma \in \sigma\left(X_{1}\right)$ and $l \in \mathbb{C} \backslash \sigma\left(X_{2}\right)$. 
Now we will apply the Stieltjes inversion formula to $f(l, \gamma)$ in order to recover the principal function $g(\delta, \gamma)$ of $T$. Since we have $\lim _{\epsilon \searrow 0} q\left(\frac{\delta}{\left\|v_{2}\right\|}+i \frac{\epsilon}{\left\|v_{2}\right\|}\right)=\zeta\left(\frac{\delta}{\left\|v_{2}\right\|}\right)$ for $\delta \in \sigma\left(X_{2}\right)$ as in 15, we get

$$
\begin{aligned}
g(\delta, \gamma)= & \frac{1}{\pi} \lim _{\epsilon \searrow 0} \operatorname{Im} f(\delta+i \epsilon, \gamma) \\
= & \frac{1}{\pi} \operatorname{Arg}\left(1-\frac{\bar{\alpha}}{\left\|v_{1}\right\|\left\|v_{2}\right\|} \zeta\left(\frac{\gamma}{\left\|v_{1}\right\|}\right) \zeta\left(\frac{\delta}{\left\|v_{2}\right\|}\right)\right)+\frac{1}{\pi} \operatorname{Arg}\left(1-\frac{\alpha}{\left\|v_{1}\right\|\left\|v_{2}\right\|} \zeta \overline{\left(\frac{\gamma}{\left\|v_{1}\right\|}\right)} \zeta\left(\frac{\delta}{\left\|v_{2}\right\|}\right)\right) \\
& -\frac{1}{\pi} \operatorname{Arg}\left(1-\frac{\alpha}{\left\|v_{1}\right\|\left\|v_{2}\right\|} \zeta\left(\frac{\gamma}{\left\|v_{1}\right\|}\right) \zeta\left(\frac{\delta}{\left\|v_{2}\right\|}\right)\right)-\frac{1}{\pi} \operatorname{Arg}\left(1-\frac{\bar{\alpha}}{\left\|v_{1}\right\|\left\|v_{2}\right\|} \zeta\left(\frac{\gamma}{\left\|v_{1}\right\|}\right) \zeta\left(\frac{\delta}{\left\|v_{2}\right\|}\right)\right) .
\end{aligned}
$$

Setting $\frac{\alpha}{\left\|v_{1}\right\|\left\|v_{2}\right\|}=r e^{i \phi}(0<r<1), \zeta\left(\frac{\gamma}{\left\|v_{1}\right\|}\right)=e^{i \theta_{1}}$, and $\zeta\left(\frac{\delta}{\left\|v_{2}\right\|}\right)=e^{i \theta_{2}}$, we get

$$
\begin{gathered}
g(\delta, \gamma)=\frac{1}{\pi}\left(\operatorname{Arg}\left(1-r e^{i\left(-\phi+\theta_{1}+\theta_{2}\right)}\right)+\operatorname{Arg}\left(1-r e^{i\left(\phi-\theta_{1}+\theta_{2}\right)}\right)\right. \\
\left.-\operatorname{Arg}\left(1-r e^{i\left(\phi+\theta_{1}+\theta_{2}\right)}\right)-\operatorname{Arg}\left(1-r e^{i\left(-\phi-\theta_{1}+\theta_{2}\right)}\right)\right) \\
=\frac{1}{\pi}\left(\arctan \left(\frac{-r \sin \left(-\phi+\left(\theta_{1}+\theta_{2}\right)\right)}{1-r \cos \left(-\phi+\left(\theta_{1}+\theta_{2}\right)\right.}\right)+\arctan \left(\frac{-r \sin \left(\phi-\left(\theta_{1}-\theta_{2}\right)\right)}{1-r \cos \left(\phi-\left(\theta_{1}-\theta_{2}\right)\right)}\right)\right. \\
\left.\quad-\arctan \left(\frac{-r \sin \left(\phi+\left(\theta_{1}+\theta_{2}\right)\right)}{1-r \cos \left(\phi+\left(\theta_{1}+\theta_{2}\right)\right)}\right)-\arctan \left(\frac{-r \sin \left(-\phi-\left(\theta_{1}-\theta_{2}\right)\right)}{1-r \cos \left(-\phi-\left(\theta_{1}-\theta_{2}\right)\right)}\right)\right) \\
=\frac{1}{\pi}\left(\arctan \left(\frac{r \sin \left(\phi-\left(\theta_{1}+\theta_{2}\right)\right)}{1-r \cos \left(\phi-\left(\theta_{1}+\theta_{2}\right)\right)}\right)+\arctan \left(\phi+\left(\theta_{1}+\theta_{2}\right)\right)\right. \\
\left.-\arctan \left(\frac{r \sin \left(\phi-\left(\theta_{1}-\theta_{2}\right)\right)}{1-r \cos \left(\phi-\left(\theta_{1}-\theta_{2}\right)\right)}\right)-\arctan \left(\frac{r \sin \left(\phi+\left(\theta_{1}-\theta_{2}\right)\right)}{1-r \cos \left(\phi+\left(\theta_{1}-\theta_{2}\right)\right)}\right)\right) .
\end{gathered}
$$

\section{On THE ESSENTIAL SPECTRUM}

As an application, we determine the essential spectrum of the operator $T$ whose principal function we found in Section 2. We will use the following, which follows from Theorem 8.1 of [4]:

Theorem $3.1(\sqrt[4]{4})$. Suppose $T$ is an operator on a Hilbert space $\mathcal{H}$ with self-commutator $T^{*} T-T T^{*}$ in trace class. For $\gamma+i \delta$ not in the essential spectrum of $T$,

$$
g(\delta, \gamma)=\operatorname{ind}(T-(\gamma+i \delta))
$$

where $g(\delta, \gamma)$ is the principal function for $T$.

Lemma 3.2. Let $0<r<1$ and let

$$
\begin{aligned}
& h\left(r, \phi, \theta_{1}, \theta_{2}\right) \\
&=\frac{1}{\pi}\left(\arctan \left(\frac{r \sin \left(\phi-\left(\theta_{1}+\theta_{2}\right)\right)}{1-r \cos \left(\phi-\left(\theta_{1}+\theta_{2}\right)\right)}\right)+\arctan \left(\frac{r \sin \left(\phi+\left(\theta_{1}+\theta_{2}\right)\right)}{1-r \cos \left(\phi+\left(\theta_{1}+\theta_{2}\right)\right)}\right)\right. \\
&\left.\quad-\arctan \left(\frac{r \sin \left(\phi-\left(\theta_{1}-\theta_{2}\right)\right)}{1-r \cos \left(\phi-\left(\theta_{1}-\theta_{2}\right)\right)}\right)-\arctan \left(\frac{r \sin \left(\phi+\left(\theta_{1}-\theta_{2}\right)\right)}{1-r \cos \left(\phi+\left(\theta_{1}-\theta_{2}\right)\right)}\right)\right) .
\end{aligned}
$$

(a) If $\phi=0$ or $\phi=\pi$, then $h\left(r, \phi, \theta_{1}, \theta_{2}\right)=0$.

(b) If $0<\phi<\pi$, then for all $\theta_{1}, \theta_{2} \in[-\pi, 0]$, we have

$$
-\frac{2}{\pi} \arctan \left(\frac{2 r \sin \phi}{1-r^{2}}\right) \leq h\left(r, \phi, \theta_{1}, \theta_{2}\right) \leq 0
$$


with equality holding on the left when $\theta_{1}=\theta_{2}=-\frac{\pi}{2}$ and equality holding on the right only when $\theta_{1} \in\{-\pi, 0\}$ or $\theta_{2} \in\{-\pi, 0\}$.

(c) If $\pi<\phi<2 \pi$, then for all $\theta_{1}, \theta_{2} \in[-\pi, 0]$, we have

$$
0 \leq h\left(r, \phi, \theta_{1}, \theta_{2}\right) \leq-\frac{2}{\pi} \arctan \left(\frac{2 r \sin \phi}{1-r^{2}}\right),
$$

with equality holding on the right when $\theta_{1}=\theta_{2}=-\frac{\pi}{2}$ and equality holding on the left only when $\theta_{1} \in\{-\pi, 0\}$ or $\theta_{2} \in\{-\pi, 0\}$.

Proof. Part (a) is clear and we may assume $\phi \in(0, \pi) \cup(\pi, 2 \pi)$.

Let $\nu=\theta_{1}+\theta_{2}$ and $\mu=\theta_{1}-\theta_{2}$. Then we are interested in the function

$$
\begin{gathered}
\tilde{h}(r, \phi, \nu, \mu)=\frac{1}{\pi}\left(\arctan \left(\frac{r \sin (\phi-\nu)}{1-r \cos (\phi-\nu)}\right)+\arctan \left(\frac{r \sin (\phi+\nu)}{1-r \cos (\phi+\nu)}\right)\right. \\
\left.-\arctan \left(\frac{r \sin (\phi-\mu)}{1-r \cos (\phi-\mu)}\right)-\arctan \left(\frac{r \sin (\phi+\mu)}{1-r \cos (\phi+\mu)}\right)\right),
\end{gathered}
$$

where

$$
\begin{gathered}
-2 \pi \leq \nu \leq 0 \\
-\min (-\nu, 2 \pi+\nu) \leq \mu \leq \min (-\nu, 2 \pi+\nu) .
\end{gathered}
$$

In particular, we always have $|\mu| \leq \pi$. Note that the boundaries of the region described by (19) -20 ) correspond to $\theta_{1} \in\{-\pi, 0\}$ or $\theta_{2} \in\{-\pi, 0\}$, where the function $h$ vanishes.

An extreme point of $\tilde{h}$ not on the boundary can occur only where

$$
\frac{\partial \tilde{h}}{d \nu}=\frac{\partial \tilde{h}}{d \mu}=0
$$

We compute

$$
\frac{d}{d x} \arctan \left(\frac{r \sin (x)}{1-r \cos (x)}\right)=\frac{r(\cos (x)-r)}{1-2 r \cos (x)+r^{2}}
$$

We also compute

$$
\frac{d}{d c}\left(\frac{c-r}{1-2 r c+r^{2}}\right)=\frac{1-r^{2}}{\left(1-2 r c+r^{2}\right)^{2}}>0,
$$

so the function

$$
c \mapsto \frac{r(c-r)}{1-2 r c+r^{2}}
$$

is strictly increasing on $[-1,1]$. Therefore,

$$
\begin{aligned}
\frac{\partial \tilde{h}}{d \nu}=\frac{d}{d \nu}\left(\arctan \left(\frac{r \sin (\phi-\nu)}{1-r \cos (\phi-\nu)}\right)+\arctan (\right. & \left.\left.\frac{r \sin (\phi+\nu)}{1-r \cos (\phi+\nu)}\right)\right) \\
& =\frac{-r(\cos (\phi-\nu)-r)}{1-2 r \cos (\phi-\nu)+r^{2}}+\frac{r(\cos (\phi+\nu)-r)}{1-2 r \cos (\phi+\nu)+r^{2}}
\end{aligned}
$$

vanishes if and only if $\cos (\phi-\nu)=\cos (\phi+\nu)$, which in turn occurs if and only if either $\nu \in \pi \mathbf{Z}$ or $\phi \in \pi \mathbf{Z}$. We assumed $\phi \notin \pi \mathbf{Z}$. If $\nu \in\{-2 \pi, 0\}$, then $\nu$ is on the boundary of the interval (19), so the only possibility that is not on the boundary of the region is $\nu=-\pi$.

Arguing as above, $\frac{\partial \tilde{h}}{d \mu}=0$ if and only if $\cos (\phi-\mu)=\cos (\phi+\mu)$. Avoiding the boundary, this leaves only $\mu=0$. We conclude that the only extreme point of $\tilde{h}$ not on the boundary occurs at $(\nu, \mu)=(-\pi, 0)$, i.e., at $\left(\theta_{1}, \theta_{2}\right)=\left(-\frac{\pi}{2},-\frac{\pi}{2}\right)$, and the value of $\tilde{h}$ there is

$$
-\frac{2}{\pi}\left(\arctan \left(\frac{r \sin \phi}{1-r \cos \phi}\right)+\arctan \left(\frac{r \sin \phi}{1+r \cos \phi}\right)\right) \text {. }
$$


We have the identity, for $\alpha, \beta \in \mathbb{R}$,

$$
\arctan (\alpha)+\arctan (\beta) \in \arctan \left(\frac{\alpha+\beta}{1-\alpha \beta}\right)+\pi \mathbf{Z} .
$$

Letting

$$
\alpha=\frac{r \sin \phi}{1-r \cos \phi} \quad \text { and } \quad \beta=\frac{r \sin \phi}{1+r \cos \phi},
$$

since

$$
0<\alpha \beta=\frac{r^{2} \sin ^{2} \phi}{1-r^{2}+r^{2} \sin ^{2} \phi}<1,
$$

we find that the quantity 21 equals

$$
-\frac{2}{\pi} \arctan \left(\frac{\alpha+\beta}{1-\alpha \beta}\right)=-\frac{2}{\pi} \arctan \left(\frac{2 r \sin \phi}{1-r^{2}}\right) .
$$

We already observed that on the boundaries of the region described by $(19)-(20)$, the function $\tilde{h}$ vanishes and we just showed that the only extreme value not on the boundary is $(22)$, which is attained when $\theta_{1}=\theta_{2}=-\frac{\pi}{2}$. In particular, $\tilde{h}$ is never vanishing on the interior of the region. This completes the proof of (b) and (c).

Theorem 3.3. Let $T=l\left(v_{1}\right)+l\left(v_{1}\right)^{*}+i\left(r\left(v_{2}\right)+r\left(v_{2}\right)^{*}\right)$ with $v_{1}$ and $v_{2}$ linearly independent and $\operatorname{Im}\left\langle v_{1}, v_{2}\right\rangle \neq 0$. Then the essential spectrum $\sigma_{e}(T)$ of $T$ is the closed rectangle

$$
\left\{\gamma+i \delta \in \mathbb{C}|| \gamma \mid \leq 2\left\|v_{1}\right\| \text { and }|\delta| \leq 2\left\|v_{2}\right\|\right\},
$$

which equals the spectrum $\sigma(T)$ of $T$.

Proof. By Lemma 2.1. we have that $\sigma(T)$ is contained in the rectangle 23). For $\gamma \in \sigma\left(X_{1}\right)$ and $\delta \in \sigma\left(X_{2}\right)$, we have the formula of the principal function $g(\delta, \gamma)$ in (18). By Lemma 3.2, $-1<g(\delta, \gamma) \leq 0$ if $\operatorname{Im}\left\langle v_{1}, v_{2}\right\rangle>0$, and $0 \leq g(\delta, \gamma)<1$ if $\operatorname{Im}\left\langle v_{1}, v_{2}\right\rangle<0$. The equality $g(\delta, \gamma)=0$ holds only when $\gamma \in\left\{2\left\|v_{1}\right\|,-2\left\|v_{1}\right\|\right\}$ or $\delta \in\left\{2\left\|v_{2}\right\|,-2\left\|v_{2}\right\|\right\}$, i.e., when $\gamma$ and $\delta$ are on the boundary of the rectangle (23). So the function $g(\delta, \gamma)$ does not assume any integer value on the interior of the rectangle. But, by Theorem 3.1. if $\gamma+i \delta \notin \sigma_{e}(T)$, then $g(\delta, \gamma)=\operatorname{ind}(T-(\gamma+i \delta))$. So the whole interior of the rectangle is included in the essential spectrum of $T$. Since $\sigma_{e}(T)$ is closed in $\mathbb{C}$ and is contained in $\sigma(T)$, we have $\sigma_{e}(T)$ equals the rectangle 23 .

Proposition 3.4 ([1]). Suppose that $T$ has compact self-commutator $T^{*} T-T T^{*}$ on a Hilbert space $\mathcal{H}$ and $\operatorname{ind}(T-\lambda)=0$ for all $\lambda \in \mathbb{C} \backslash \sigma_{e}(T)$. Then $T$ is of the form $N+K$ where $N$ is normal and $K$ is compact.

Corollary 3.5. The operator $T=l\left(v_{1}\right)+l\left(v_{1}\right)^{*}+i\left(r\left(v_{2}\right)+r\left(v_{2}\right)^{*}\right)$ with linearly independent $v_{1}$ and $v_{2}$ and $\operatorname{Im}\left\langle v_{1}, v_{2}\right\rangle \neq 0$ is normal plus compact.

Example 3.6. Let $v_{2}$ and $u$ be orthogonal vectors in a Hilbert space $\mathcal{H}$ with $\left\|v_{2}\right\|=\|u\|=1$, and let $\alpha=\frac{1}{\sqrt{2}}-\frac{1}{\sqrt{2}} i \in \mathbb{C}$. Set $v_{1}$ in $\mathcal{H}$ by $v_{1}=\alpha v_{2}+u$. Suppose that $T$ is a bounded operator on the full Fock space $\mathcal{F}(\mathcal{H})$ defined by $T=l\left(v_{1}\right)+l\left(v_{1}\right)^{*}+i\left(r\left(v_{2}\right)+r\left(v_{2}\right)^{*}\right)$. Then, $\left[T, T^{*}\right]=2 \sqrt{2} P$ and it is an one-dimensional projection on $\mathcal{F}(\mathcal{H})$. So, by restricting $T^{*}$ to its pure part $\overline{\operatorname{alg}\left(T, T^{*}, 1\right) \Omega}, T^{*}$ is a completely non-normal hyponormal operator.

We can find the principal function $g(\delta, \gamma)$ of $T$ by the formula (18). For each pair $(\delta, \gamma)$ such that $|\delta| \leq 2$ and $|\gamma| \leq 2 \sqrt{2}$, we have

$$
\begin{aligned}
g(\delta, \gamma)=\frac{1}{\pi} \operatorname{Arg}(1 & \left.-\left(\frac{1}{2}+\frac{1}{2} i\right) \zeta\left(\frac{\gamma}{\sqrt{2}}\right) \zeta(\delta)\right)+\frac{1}{\pi} \operatorname{Arg}\left(1-\left(\frac{1}{2}-\frac{1}{2} i\right) \overline{\zeta\left(\frac{\gamma}{\sqrt{2}}\right)} \zeta(\delta)\right) \\
& \left.-\frac{1}{\pi} \operatorname{Arg}\left(1-\left(\frac{1}{2}-\frac{1}{2} i\right) \zeta\left(\frac{\gamma}{\sqrt{2}}\right) \zeta(\delta)\right)-\frac{1}{\pi} \operatorname{Arg}\left(1-\left(\frac{1}{2}+\frac{1}{2} i\right) \zeta \overline{\left(\frac{\gamma}{\sqrt{2}}\right)} \zeta(\delta)\right)\right)
\end{aligned}
$$


where $\zeta(t)=\frac{t-i \sqrt{4-t^{2}}}{2}$ for $t \in[-2,2]$. Since $\operatorname{Im}\left\langle v_{1}, v_{2}\right\rangle<0$, we have $0 \leq g(\delta, \gamma)<1$ for all $(\delta, \gamma) \in \mathbb{R}^{2}$. By Lemma $3.2, g(\delta, \gamma)$ is vanishing only when $(\delta, \gamma)$ is on the boundary of the rectangle $\left\{(\delta, \gamma) \in \mathbb{R}^{2}|| \gamma \mid \leq\right.$ $2 \sqrt{2}$ and $|\delta| \leq 2\}$. Therefore, $\sigma(T)=\sigma_{e}(T)=\{\gamma+i \delta \in \mathbb{C}|| \gamma \mid \leq 2 \sqrt{2}$ and $|\delta| \leq 2\}$. See Figure 1 .

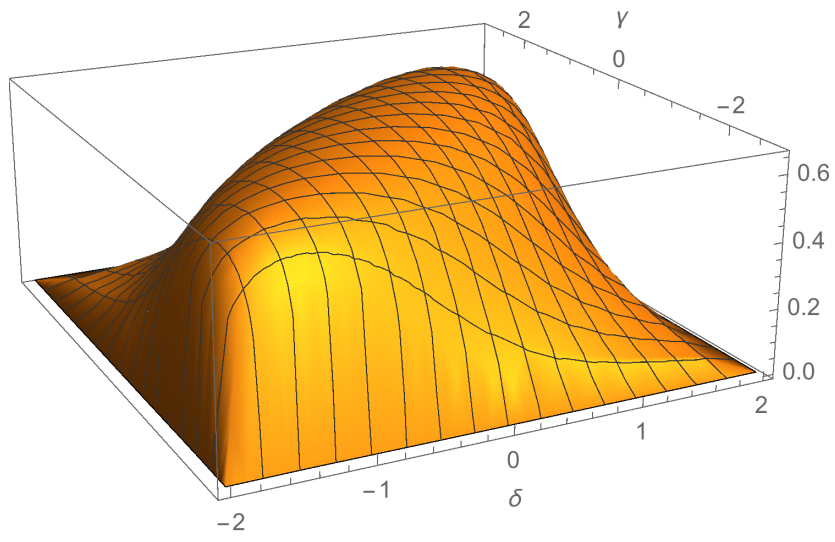

Figure 1. The principal function $g(\delta, \gamma)$ of T where $v_{1}=\alpha v_{2}+u, \alpha=\frac{1}{\sqrt{2}}-\frac{1}{\sqrt{2}} i, u \perp v_{1}$ and $\left\|v_{2}\right\|=\|u\|=1$.

\section{REFERENCES}

[1] L. G. Brown, R. G. Douglas, and P. A. Fillmore, Unitary equivalence modulo the compact operators and extensions of $C^{*}$-algebras, Proceedings of a Conference on Operator Theory (Dalhousie Univ., Halifax, N.S., 1973), Springer, Berlin, 1973, pp. 58-128. Lecture Notes in Math., Vol. 345.

[2] J. B. Conway and P. McGuire, Operators with $C^{*}$-algebra generated by a unilateral shift, Trans. Amer. Math. Soc. 284 (1984), no. 1, 153-161.

[3] R. W. Carey and J. D. Pincus, An invariant for certain operator algebras, Proc. Nat. Acad. Sci. U.S.A. 71 (1974), $1952-1956$

[4] - Mosaics, principal functions, and mean motion in von Neumann algebras, Acta Math. 138 (1977), no. 3-4, $153-218$.

[5] _ Construction of seminormal operators with prescribed mosaic, Indiana Univ. Math. J. 23 (1973/74), 1155-1165.

[6] M. Martin and M. Putinar, Lectures on hyponormal operators, Operator Theory: Advances and Applications, vol. 39, Birkhäuser Verlag, Basel, 1989.

[7] D. Voiculescu, Free probability for pairs of faces I, Comm. Math. Phys. 332 (2014), no. 3, 955-980.

[8] __ Free probability for pairs of faces II: 2-variables bi-free partial $R$-transform and systems with rank $\leq 1$ commutation, preprint, available at arXiv:1308.2035

K. Dykema, Department of Mathematics, Texas A\&M University, College Station, TX 77843-3368, USA

E-mail address: kdykema@math.tamu.edu

W. Na, Department of Mathematics, Texas A\&M University, College Station, TX 77843-3368, USA

E-mail address: wonhee@math.tamu.edu 